\title{
The MICE Demonstration of Muon Ionization Cooling
}

\author{
Tanaz Angelina Mohayai ${ }^{* \dagger}$ \\ Illinois Institute of Technology \\ E-mail: tmohayai@hawk.iit.edu
}

\begin{abstract}
Muon beams of low emittance provide the basis for intense, well characterised neutrino beams at Neutrino Factory and lepton-antilepton collisions up to several $\mathrm{TeV}$ at Muon Collider. The international Muon Ionization Cooling Experiment, MICE will demonstrate muon ionization cooling, the technique proposed to reduce the phase-space volume occupied by the muon beam at such facilities. To obtain sustainable ionization cooling, the muon beam loses transverse and longitudinal momentum via ionization energy loss in material (absorber) and then has the longitudinal momentum restored in an RF cavity. The combined effect is to reduce the transverse emittance of the beam. The design of the cooling demonstration experiment will be presented together with a summary of the performance of each of its components.
\end{abstract}

38th International Conference on High Energy Physics

3-10 August 2016

Chicago, USA

\footnotetext{
* Speaker.

$\dagger$ This paper is written on behalf of the MICE collaboration. MICE is supported by DOE, INFN, and STFC. The first author would like to thank the supports of DOE Office of Science Graduate Student Research (SCGSR) program under contract No. DE-AC05-06OR23100 and IIT Irwin Fieldhouse Fellowship.
} 


\section{Muon Ionization Cooling Principle}

Low emittance muon beams are essential to future Neutrino Factory (NF) and Muon Collider (MC) facilities. In NF, muon beams produce intense and pure two-flavour neutrino beams. Muon beams in MC lose less energy through synchrotron radiation compared with electron beams in an electron-positron machine. This makes MC more compact and cost effective than an electronpositron machine [1]. The muon beams at the front end of NF or MC are produced through the decay of pions, and the pions in turn are produced when high-power proton beams bombard a target. However, these pion-decay muons occupy a large phase-space volume and to optimize muon yield and fit the muon beam into the small apertures of a future muon facility, the phase-space volume or the beam emittance needs to be reduced [2]. The process of reducing beam emittance is known as beam cooling. Typical muon beams produced via pion decay have emittance ranges of $15-20 \pi$ $\mathrm{mm} \cdot \mathrm{rad}$ at the front end of NF or MC. The desired emittance range of the muon beam at NF is 2-5 $\pi \mathrm{mm} \cdot \mathrm{rad}$. MC needs further cooling with a desired transverse emittance of $0.025 \pi \mathrm{mm} \cdot \mathrm{rad}$ and longitudinal emittance of $72 \pi \mathrm{mm} \cdot \mathrm{rad}$ [3]. The short muon lifetime makes the traditional cooling techniques such as synchrotron radiation and stochastic cooling inefficient. Ionization cooling is the only feasable cooling technique for reducing the muon beam emittance within the short muon lifetime and is made possible by the muon's long interaction length [2]. In the process of muon ionization cooling, the muon's transverse and longitudinal momentum is reduced via ionization energy loss in an absorber. For sustainable ionization cooling, the longitudinal momentum is restored by subsequently passing the muon beam through a Radio Frequency (RF) cavity [4]. The process of cooling is complicated by the simultaneous multiple Coulomb scattering process which increases the emittance of the beam [2]. The rate of change of the normalized transverse emittance of a muon beam which traverses the material along the beam axis, $z$ is:

$$
\frac{d \varepsilon_{\perp}}{d z} \simeq-\frac{\varepsilon_{\perp}}{\beta^{2} E_{\mu}}\left\langle\frac{d E}{d z}\right\rangle+\frac{\beta_{\perp}(13.6 \mathrm{MeV})^{2}}{2 \beta^{3} E_{\mu} m_{\mu} X_{0}}
$$

where $\beta, E_{\mu}$, and $m_{\mu}$ are the muon velocity, energy, and mass, $\frac{d E}{d z}$ the magnitude of the mean energy loss rate through ionization, $X_{0}$ the absorber radiation length, and $\beta_{\perp}$ the transverse betatron function or beta at the absorber. The first term in Eq. 1.1 describes ionization cooling via energy loss and the second term heating due to multiple Coulomb scattering. As the muon beam travels along the cooling channel, its rates of cooling and heating respectively decrease and increase; this process continues until the heating term and the cooling terms are equal, in which case the cooling channel is said to have reached "equilibrium emittance" [2]. Setting the rate of change of normalized transverse emittance to zero yields the equilibrium emittance [5],

$$
\varepsilon_{\perp} \cong \frac{\beta_{\perp}(13.6 \mathrm{MeV})^{2}}{2 X_{0} \beta m_{\mu}}\left\langle\frac{d E}{d z}\right\rangle^{-1}
$$

A smaller equilibrium emittance results in a more effective emittance reduction, which from the above equation is interpreted as a minimized $\beta_{\perp}$ and a maximized $X_{0}$, and $\frac{d E}{d z}$. In MICE, a small beta function is achieved by focusing the beam tightly at the absorber by the use of a solenoid focusing channel. In addition, the use of low- $Z$ absorbing materials such as $\mathrm{LiH}$ (lithium hydride) and $\mathrm{LH}_{2}$ (liquid hydrogen) leads to large radiation length and energy loss. 


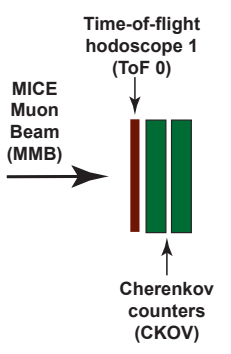

MICE

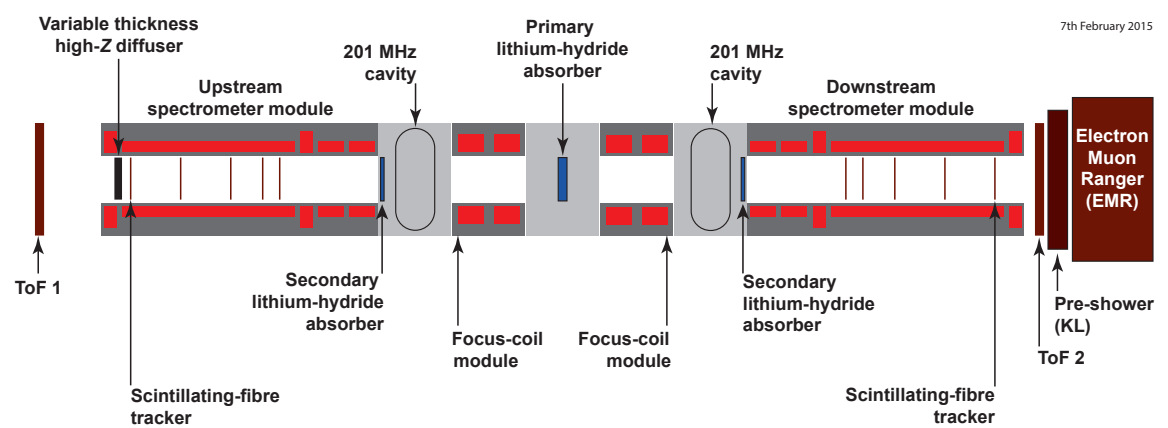

Figure 1: Schematic diagram of the final MICE cooling demonstration step.

\section{Cooling Demonstration Lattice}

In the MICE lattice studied in this paper, a realistic muon beam has been simulated using the MICE Analysis User Software, MAUS. MAUS simulates the particle interactions in MICE using Geant 4 , reconstructs hits and tracks from the detectors and provides the framework for accelerator physics analysis [6]. The plot of the transverse beta function evolution along the MICE lattice is shown in Fig. 2 and it displays the transverse beta functions for various momentum values of 140 $\mathrm{MeV} / c, 200 \mathrm{MeV} / c$, and $240 \mathrm{MeV} / c$. The initial beam emittance is kept at a constant value of $6 \pi$ $\mathrm{mm} \cdot \mathrm{rad}$ throughout this paper. The beta is smallest at the location of the central $\mathrm{LiH}$ absorber. In contrast, the beam size or beta is largest in the center of the Absorber Focus Coil, AFC module, which has large aperture to maximize transmission. Beta remains constant for $z$ values of $\sim \pm 4000$; these regions mark the locations of the trackers which are immersed in the uniform $4 \mathrm{~T}$ fields of the Spectrometer Solenoid modules. The muon transmission versus initial emittance plot in Fig. 2 shows that the transmission is higher for smaller beams and the largest beams have transmission of $90 \%$. The transverse emittance evolution plot in Fig. 2 shows significant emittance reduction in each absorber and a notable amount of emittance growth which is caused by non-linear effects. Overall emittance reduction is measured between the inner tracker planes and shows a reduction of 5.6\%. The fractional change in emittance versus the input emittance in Fig. 2 shows that for larger input emittance, a better overall emittance reduction can be obtained [7].

\section{Conclusion}

A revised cooling demonstration lattice was described in this paper where with the use of the existing components the performance required for a detailed study of ionization cooling was reached. Demonstration of ionization cooling in MICE sets the basis for intense and flavour-pure neutrino beams at a future Neutrino Factory and high-luminosity muon beams at a future Muon Collider. As a result, a successful completion of MICE is the essential step to establishing a new technique in particle and accelerator physics.

\section{References}

[1] D.M. Kaplan, From Neutrino Factory to Muon Collider, in proceedings of NuFact 2011, AIP (2011) [arXiv:1102.1591]. 

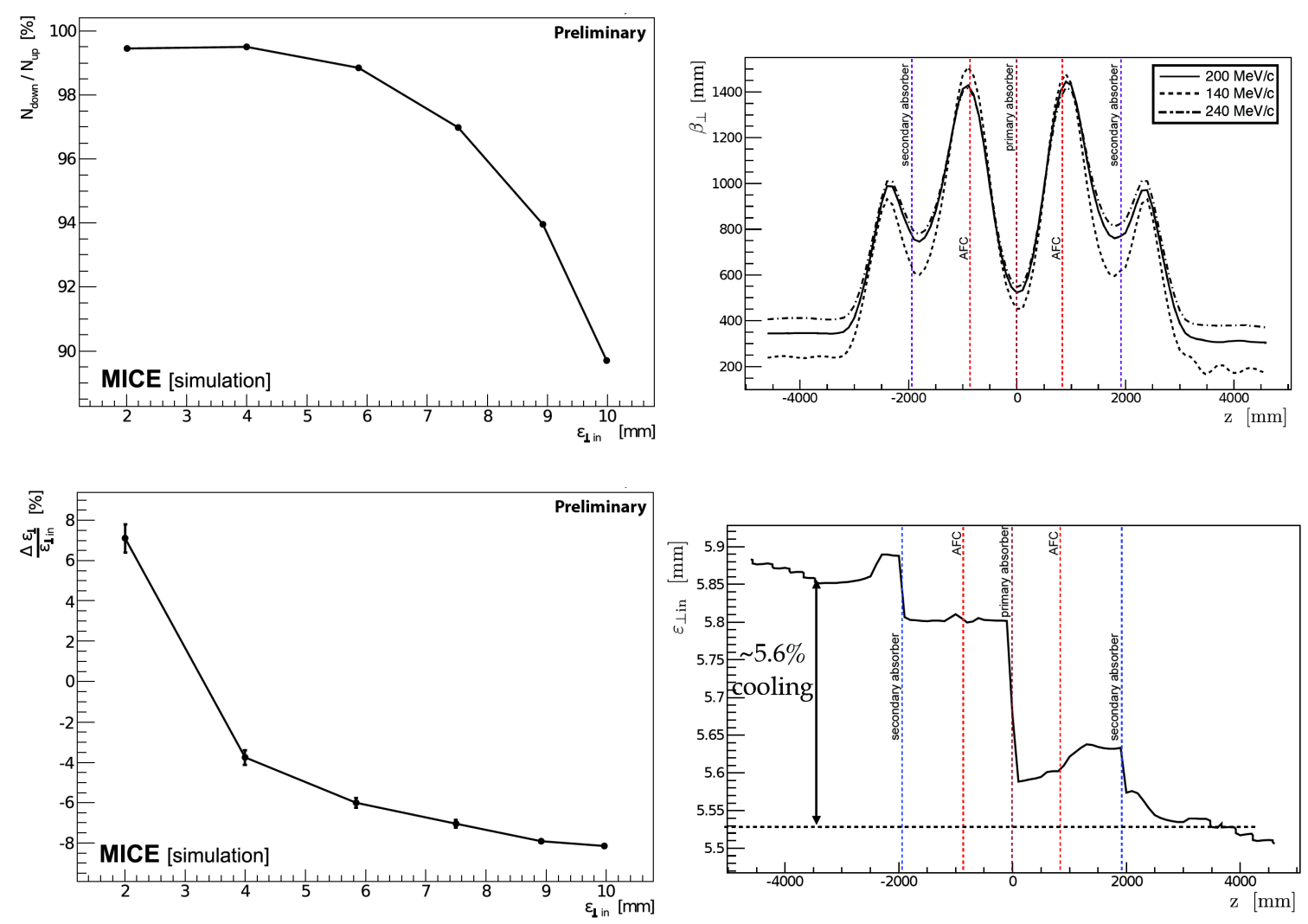

Figure 2: Muon beam transmission vs. input beam emittance for $200 \mathrm{MeV} / \mathrm{c}$ muon beam (upper left), transverse beta function evolution along the redesigned lattice (upper right), fractional change in normalized transverse emittance vs. input beam emittance for $200 \mathrm{MeV} / c$ muon beam (lower left), and transverse emittance evolution along the redesigned lattice for $200 \mathrm{MeV} / \mathrm{c}$ muon beam (lower right).

[2] R.C. Fernow, et al., Ionization Cooling, DPF/DPB New Directions in High- Energy Physics, BNL-52503, Ch. 6 (1996).

[3] R. Palmer, et al., A Complete Scheme of Ionization Cooling for a Muon Collider, in proceedings of PAC 2007 [arXiv:0711.4275] (2007).

[4] Y. Torun, et al., Installation and Commissioning of the MICE RF Module Prototype, in proceedings of PAC 2015, IPAC-2015-WEPTY055 (2015).

[5] D.V. Neuffer, Introduction to muon cooling, Nuclear Instruments and Methods in Physics Research A 532 (2004) 26-31.

[6] C.D. Tunnell, et al., MAUS: MICE Analysis User Software, in proceedings of IPAC 2011, IPAC-2011-MOPZ013 (2011).

[7] T.A. Mohayai, The MICE Demonstration of Ionization Cooling, in proceedings of COOL 2015, COOL-2015-TUPF04 (2015). 